\title{
Measurements of Dynamic Contributions to Coherent Neutron Scattering
}

\author{
Sebastian Jaksch *(D), Alexandros Koutsioubas ${ }^{(0)}$, Stefan Mattauch ${ }^{\circledR}$, Olaf Holderer \\ and Henrich Frielinghaus \\ Forschungszentrum Jülich $\mathrm{GmbH}$, Jülich Centre for Neutron Science JCNS at Heinz Maier-Leibnitz Zentrum \\ MLZ, Lichtenberstraße 1, 85747 Garching, Germany; a.koutsioumpas@fz-juelich.de (A.K.); \\ s.mattauch@fz-juelich.de (S.M.); o.holderer@fz-juelich.de (O.H.); h.frielinghaus@fz-juelich.de (H.F.) \\ * Correspondence: s.jaksch@fz-juelich.de; Tel.: +49-89-289-11-673
}

Received: 13 July 2018; Accepted: 3 August 2018; Published: 7 August 2018

\begin{abstract}
In this manuscript, we are investigating the contribution of dynamic membrane properties of phospholipid membranes to coherent scattering signals under grazing incidence. Spectroscopic measurements under grazing incidence can provide useful insight into the properties of biological membranes; however, they are often impeded by weak signals. By using grazing-incidence small-angle neutron scattering (GISANS) to identify a dynamic scattering contribution, we are able to independently corroborate the existence of a previously found dynamic mode, now measured by grazing-incidence neutron spin echo spectroscopy (GINSES). Additionally, by increasing the speed of measurement compared to GINSES from several days to hours, we were able to explore the temperature behavior of this mode in phospholipid membranes. These dynamic modes of the membranes show a wavelength of around $700 \AA$ in-plane of the membrane and are most pronounced around $37{ }^{\circ} \mathrm{C}$ and strongly decrease at lower temperatures below $25^{\circ} \mathrm{C}$ before vanishing at $20^{\circ} \mathrm{C}$. We therefore speculate that they may be linked to biologically relevant functions of the membranes themselves. To our knowledge, this is the first report of an investigation of that membrane mode by means of GISANS.
\end{abstract}

Keywords: phospholipids; membranes; GISANS; GINSES; coherent scattering; spectroscopy

\section{Introduction}

Phospholipid membranes provide a wide array of functions for cells in the form of cell membranes as they define their interface with their environment. Understanding this interface both in terms of structure and dynamics is therefore instrumental for the investigation of biological functions of cell walls. This becomes especially clear when we are considering transport mechanisms through or even along the membranes, such as diffusion along and through the them, endocytosis or function of surface proteins in the membrane. From the application side, two important examples are transport vectors for drug delivery or attack vectors for bacterial and viral pathogens.

In a previous publication, we identified a hitherto unobserved eigenmode of phospholipid membranes at physiological temperatures by means of GINSES [1]. However, due to a limited available time for the experiments, we were unable to further explore the behavior of those eigenmodes we called dynamic modes. Furthermore, since the experimental time for a single spectrum was on the order of several days, this seemed unfeasible for further investigations when there was no a priori proof of the existence of such waves for a given sample or specific sample conditions. This manuscript is the first report of these modes being measured in a GISANS experiment.

Searching for a way to overcome this, we were inspired by the method of Constantin et al. [2], where the shape of a Bragg peak scattered from lamellae was analyzed and a coherent contribution to 
the peak form was found. However, instead of focusing on the Bragg Peak and following through with the Caille theory [3], we assumed a standing wave as postulated after the GINSES measurements would contribute on its own to the coherent scattering in the identified length scale and therefore $Q$-regime.

We therefore set up a GISANS experiment where, instead of searching structural features, we optimized for resolution and signal-to-noise ratio in an area that previously was identified by the GINSES experiment. There we could indeed identify an additional scattering contribution not associated to the structure of the membrane [4].

Since the GISANS measurements are considerably faster (several hours compared to days), we were also able to perform a temperature scan that revealed a temperature response of the eigenmode of the membrane. This temperature response also allowed us to rule out instrumental artifacts and proved the thermodynamic nature of the effect.

\section{Results}

The measurements presented here were performed on the MARIA [5] instrument at MLZ. The GISANS data is shown in Figure 1 in addition to the direction of the in-plane cut used for later analyses.

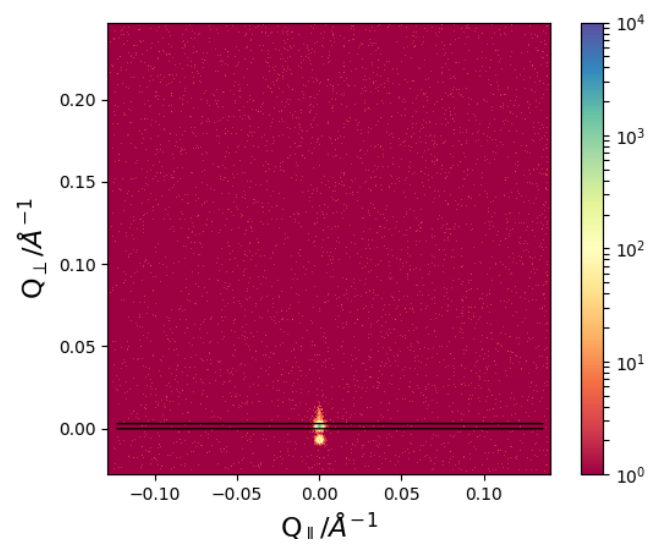

(a)

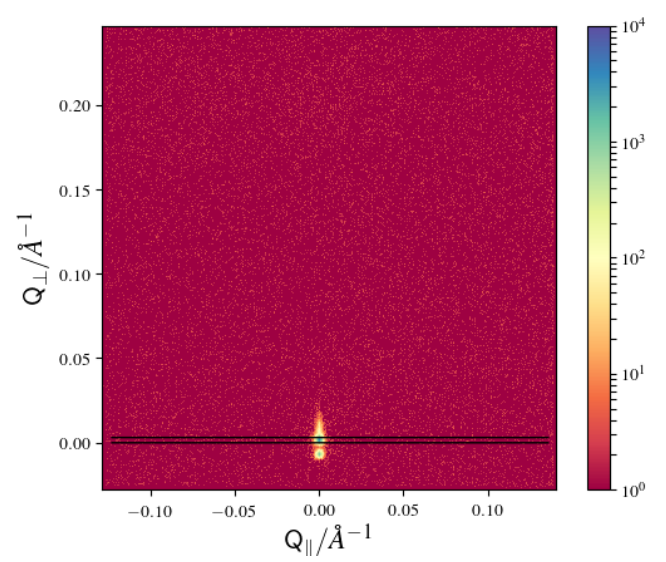

(c)

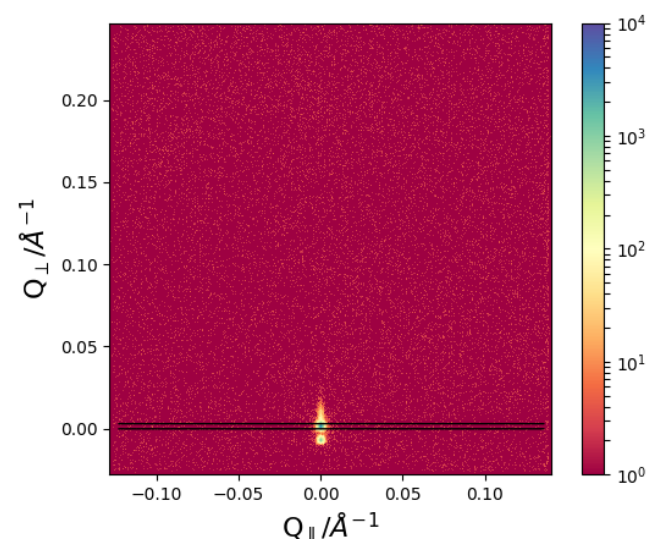

(b)

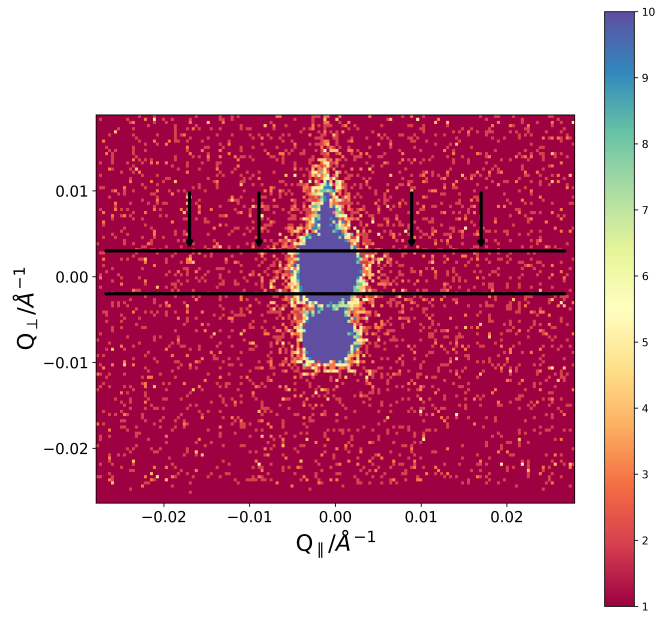

(d)

Figure 1. GISANS images at (a) $37^{\circ} \mathrm{C}$ final conditions; (b) $25^{\circ} \mathrm{C}$; (c) $20{ }^{\circ} \mathrm{C}$; and (d) $37{ }^{\circ} \mathrm{C}$ initial conditions zoom in around central area. The black lines indicate the limits for the averaging process, which are identical for all images. The vertical streak is the form-factor of the lamellae, which is not considered in this manuscript. This has been extensively discussed in one of our previous manuscripts [4]. In (d), the areas of slightly higher intensity are just visible (left hand side) as marked by the arrows. The colorscale in this case is adapted to a maximum value of 10 counts. Please note that the GISANS images are "as is" and not averaged in any way. 
The vertical cuts as indicated are then analyzed by fitting Gaussians to the appearing peaks. The corresponding data is given in Figure 2. In order to smooth the data, an average over five data points in in-plane direction is taken. This does not influence the occurrence of the described peaks, and several step width for averaging were tested in order to avoid data artifacts (see Figure $2 b$ ).

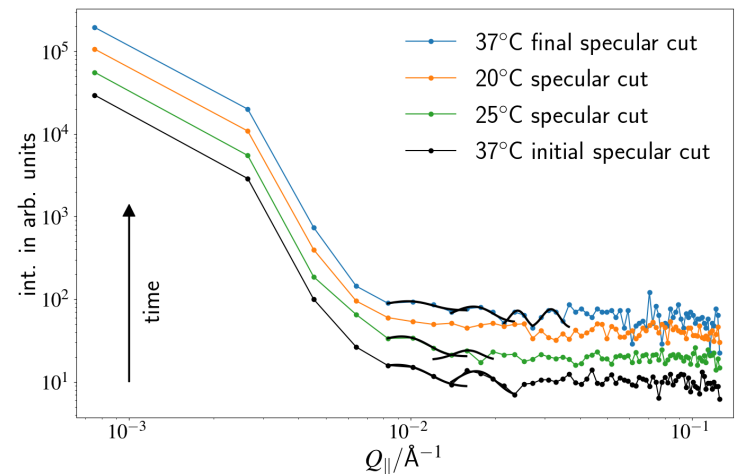

(a)

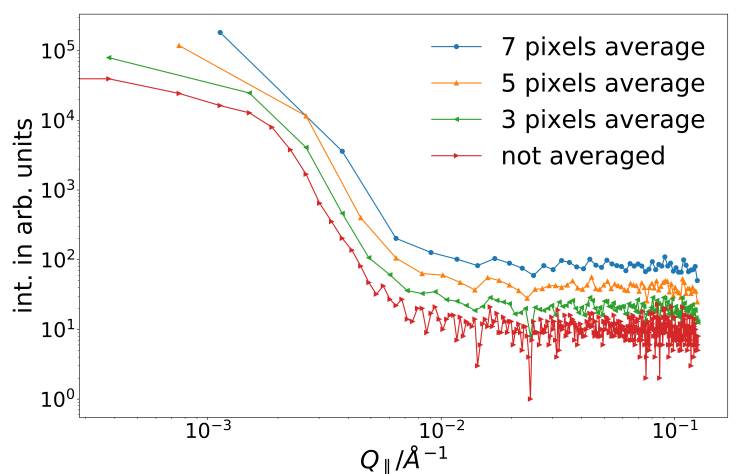

(b)

Figure 2. (a) in-plane cuts for all GISANS images. Black lines are Gaussian fits for peaks in the curves as described in the text; (b) examples for taking the average over several points in the in-plane cut. Even at no average, there are two distinct minima around the second maximum, but the data is very noisy. The first peak is still hidden in the higher intensity of the primary beam. The maxima are present in all cases, before the first one is averaged out at an average of seven points, and do not shift position. An average over five points was chosen for maximum clarity.

By inspection of the data with the naked eye, the disappearance and reappearance of the peaks can be seen. The corresponding positions for the first peak, evaluated using a Gaussian Fit, are given in Figure 3. The $Q_{\|}$-position of the first peak increases upon cooling down, corresponding to an increase in length scale, vanishes at $20^{\circ} \mathrm{C}$ and reappears when reheated to $37^{\circ} \mathrm{C}$. Even though the original value cannot be reclaimed, a return of an identical peak can be claimed since the error bars are substantial. This is also supported by the fact that this curve was achieved after the peaks completely vanished during the $20^{\circ} \mathrm{C}$ measurement on the same sample. All other results of the Gaussian fits indicated in Figure 2 are shown in Table 1.

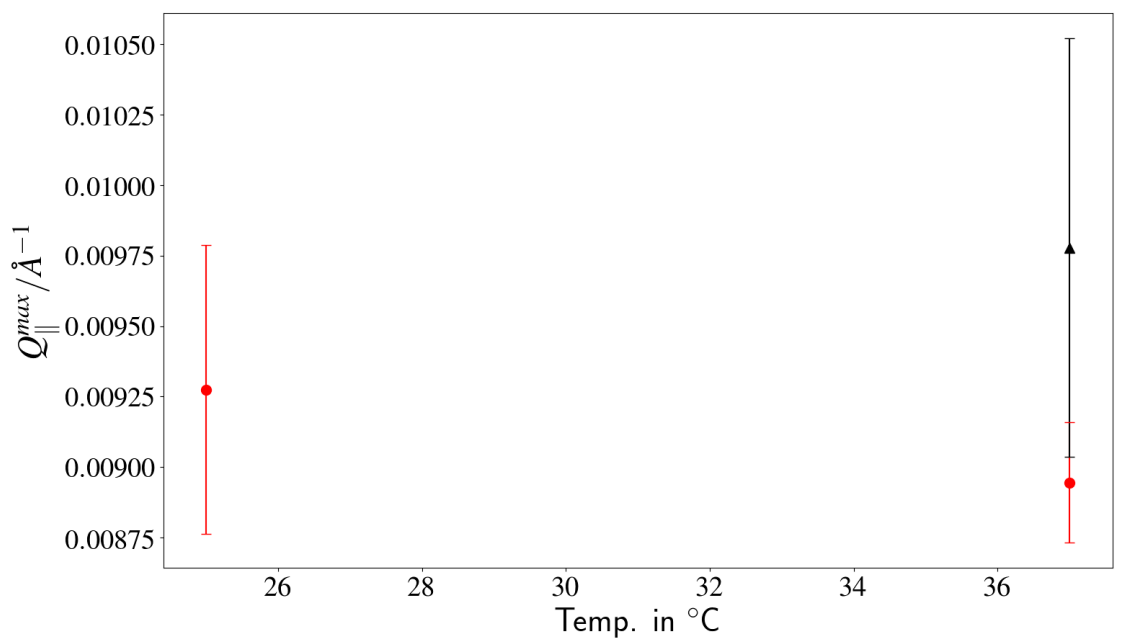

Figure 3. Position of the first peak for temperatures $37^{\circ} \mathrm{C}$ and $25^{\circ} \mathrm{C}$. The red circles are for initial conditions, the black triangle indicates the value at $37^{\circ} \mathrm{C}$ after heating up from $20^{\circ} \mathrm{C}$. 
Table 1. Results from Gaussian fits to the in-plane cuts in Figure 2. Peaks are numbered from left to right. Where the fit did not converge sufficiently to give an error $N / A$ is noted.

\begin{tabular}{ccccc}
\hline Temparature in ${ }^{\circ} \mathbf{C}$ & Peak No. & Amp. in Arb. Units & $Q_{\|}^{\max } / \AA^{-1}$ & $\sigma / \AA^{-1}$ \\
\hline 37 (initial) & 1 & $2.9 \times 10^{-2} \pm 2.4 \times 10^{-3}$ & $8.9 \times 10^{-3} \pm 2.1 \times 10^{-4}$ & $1.6 \times 10^{-3} \pm 1.5 \times 10^{-4}$ \\
37 (initial) & 2 & $3.0 \times 10^{-2} \pm 4.6 \times 10^{-3}$ & $1.7 \times 10^{-2} \pm 4.3 \times 10^{-4}$ & $1.8 \times 10^{-3} \pm 3.4 \times 10^{-4}$ \\
25 & 1 & $2.6 \times 10^{-2} \pm 6.2 \times 10^{-3}$ & $9.3 \times 10^{-3} \pm 5.1 \times 10^{-4}$ & $1.4 \times 10^{-3} \pm 4.1 \times 10^{-4}$ \\
25 & 2 & $8.6 \times 10^{-3} \pm N / A$ & $1.6 \times 10^{-2} \pm N / A$ & $1.2 \times 10^{-3} \pm N / A$ \\
37 (final) & 1 & $4.5 \times 10^{-3} \pm 3.1 \times 10^{-3}$ & $9.8 \times 10^{-3} \pm 7.4 \times 10^{-4}$ & $1.4 \times 10^{-3} \pm 7.8 \times 10^{-4}$ \\
37 (final) & 2 & $7.8 \times 10^{-3} \pm 1.4 \times 10^{-3}$ & $1.7 \times 10^{-2} \pm 4.7 \times 10^{-4}$ & $1.8 \times 10^{-3} \pm 3.8 \times 10^{-4}$ \\
37 (final) & 3 & $4.5 \times 10^{-3} \pm N / A$ & $2.4 \times 10^{-2} \pm N / A$ & $1.0 \times 10^{-2} \pm N / A$ \\
37 (final) & 4 & $7.0 \times 10^{-3} \pm 5.0 \times 10^{-4}$ & $3.2 \times 10^{-2} \pm 1.7 \times 10^{-4}$ & $1.4 \times 10^{-3} \pm 1.2 \times 10^{-4}$ \\
\hline
\end{tabular}

\section{Discussion}

The original assumption for the analysis in this manuscript was that the data presented in our previous manuscript [1] produces a coherent wave package that could be measured by static coherent, elastic neutron scattering experiments. This assumption has been proven by the measurements shown here.

However, there is more to these measurements than just the test of an assumption, since these measurements also present an independent proof in terms of method for the phenomenon observed in our previous manuscript [1]. In addition to that independent proof this also delivers a faster scanning method through other parameters, such as temperature or concentration for the existence of these wave modes, which then in turn can be used to prepare a GINSES measurement. Those measurements can then be used to extract additional data from phospholipid membrane systems, which have been hardly accessible up to now.

The length scale of the wave packages identified here is on the order of $2 \pi / Q_{\|} \approx 700 \AA$. Compared to most dynamic or quasi-elastic measurements this is quite large [6]. However, considering the usually overdamped nature of modes soft-matter systems, it is not surprising that, in membrane systems' stable modes, if they occur have to occur at a long length scale. This could also have implications on protein function and macroscopic membrane behavior since those modes link areas together that are far apart if measured in units of phospholipids (several hundreds, depending on external parameters such as surface pressure).

The disappearance and reappearance of the data also preclude any instrumental bias or artifact.

Considering the possibilities for further evaluation of the data, there is little point in analyzing amplitudes or peak shape now, since the data is just visible above background. Thus, mainly the position of the maxima is of interest, in order to identify the length scales of the wave packages. Here, further improvement of the signal-to-noise ratio may render more insights into the behavior of the system.

Another fact worth mentioning is the apparent annealing effect after cycling the temperature, when higher order peaks appear.

\section{Materials and Methods}

A full report on the experiments discussed here was published earlier [7]. Here, we describe sample preparation and instrument setup, both of which have proven to be crucial to gain reproducible data in previous experiments.

\subsection{Sample}

The sample consisted of SoyPC (L- $\alpha$-phosphatidylcholine) prepared on a polished and hydrophilically treated silicon block. The sample was obtained from Avanti Polar Lipids, Alabaster, AL, USA. In preparation for the coating of the sample on the substrate, an RCA-cleaning of the surface was performed. After that, the silicon block was rinsed with copious amounts of millipor purified water 
and then blown dry with nitrogen. The block was placed into a frame to prevent oozing-off of the sample. The sample was then dissolved in isopropanol p.A. at room temperature and shaken for $20 \mathrm{~min}$. The resulting solution was deposited on the silicon block in the horizontally aligned frame in an vacuum oven. Drying was performed over night at room temperature and $25 \mathrm{kPa}$. This ensured a slow drying that allowed for good self-assembly of the phospholipid membranes and was above the vapor pressure of the solution in order to prevent bubbling. The resulting fully dried sample (olfactory inspection) was then placed inside a closed aluminum block which was in turn filled with $\mathrm{D}_{2} \mathrm{O}$ to achieve full hydration.

The sample was then temperature controlled by a water thermostat throughout the measurements.

\subsection{Instrument Setup}

In order to be able to resolve features close to the primary beam a sample aperture of $1 \times 1 \mathrm{~mm}$ at MARIA was chosen. Under those conditions, Bragg scattering from the membranes is be negligible, due to the low intensity. However, we expected the features due to the standing wave to appear primarily around the specularly reflected beam in in-plane direction. The other collimation settings were adjusted accordingly to a $1 \times 1 \mathrm{~mm}$ slit $4.1 \mathrm{~m}$ before the sample slit and the detector is positioned $3.6 \mathrm{~m}$ behind the sample. This setup should provide an optimum in signal-to-noise ratio for GISANS at MARIA. A wavelength of $10 \AA$ and an incident angle of $0.2^{\circ}$ was used for all measurements. Each measurement lasted for $6 \mathrm{~h}$ per temperature.

\section{Conclusions}

In this manuscript, we presented the measurement of a coherent signal from a dynamic mode by means of GISANS. This both independently proved the existence of the surface modes that caused that signal as shown in our previous manuscript [1] as well as allows for a faster scanning for several parameters.

The surface modes have a structure size of approximately $700 \AA$ and may contribute to membrane stability and membrane function, since they allow the dissipation of energy in the membrane and at the same time correlate areas of the membrane that are several hundred phospholid units apart. In addition to our previous measurements, a temperature dependence was also discovered, where the dynamic modes decrease at lower temperatures, and eventually vanish below $20^{\circ} \mathrm{C}$. Here, it should be noted that the signal might simply be suppressed by background scattering.

Author Contributions: Conceptualization, S.J., O.H. and H.F.; Methodology, A.K. and S.J.; Software, S.J.; Analysis, S.J.; Writing—Original Draft Preparation, S.J.; Writing—Review and Editing, all authors.

Funding: This research received no external funding.

Acknowledgments: The authors gratefully acknowledge the granting of beam-time by the Heinz Maier-Leibnitz Centre (MLZ), Garching, Germany.

Conflicts of Interest: The authors declare no conflict of interest.

\section{References}

1. Jaksch, S.; Holderer, O.; Gvaramia, M.; Ohl, M.; Monkenbusch, M.; Frielinghaus, H. Nanoscale rheology at solid-complex fluid interfaces. Sci. Rep. 2017, 7, 4417. [CrossRef] [PubMed]

2. Constantin, D.; Brotons, G.; Salditt, T.; Freyssingeas, É.; Madsen, A. Dynamics of bulk fluctuations in a lamellar phase studied by coherent X-ray scattering. Phys. Rev. E 2006, 74, 031706. [CrossRef] [PubMed]

3. Caille, A. Remarques sur la diffusion des rayons $X$ dans les smectiques. CR Acad. Sci. Ser. B 1972, 274, 891-893.

4. Jaksch, S.; Lipfert, F.; Koutsioubas, A.; Mattauch, S.; Holderer, O.; Ivanova, O.; Frielinghaus, H.; Hertrich, S.; Fischer, S.F.; Nickel, B. Influence of ibuprofen on phospholipid membranes. Phys. Rev. E 2015, 91, 022716. [CrossRef] [PubMed] 
5. Mattauch, S.; Koutsioubas, A.; Rücker, U.; Korolkov, D.; Fracassi, V.; Daemen, J.; Schmitz, R.; Bussmann, K.; Suxdorf, F.; Wagener, M.; et al. The high-intensity reflectometer of the Jülich Centre for Neutron Science: MARIA. J. Appl. Crystallogr. 2018, 51, 646-654. [CrossRef] [PubMed]

6. Fragneto, G.; Rheinstädter, M. Structural and dynamical studies from bio-mimetic systems: An overview. C. R. Phys. 2007, 8, 865-883. [CrossRef]

7. Jaksch, S.; Koutsioubas, A.; Mattauch, S.; Holderer, O.; Frielinghaus, H. Preliminary Report on Measurements of Dynamic Contributions to Coherent Neutron Scattering. arXiv 2018, arXiv:1803.11041.

(C) 2018 by the authors. Licensee MDPI, Basel, Switzerland. This article is an open access article distributed under the terms and conditions of the Creative Commons Attribution (CC BY) license (http:// creativecommons.org/licenses/by/4.0/). 